Cómo citar este trabajo: Cordero Ramos, N. y Cáceres Feria, R. (2020) Mujeres africanas en Situación de Trata: Diversidades, resistencias y oportunidades. Revista del Laboratorio Iberoamericano para el Estudio Sociohistórico de las Sexualidades, 04 art. 10, $192-206$. https://doi.org/10.46661/ relies.5103

\title{
Mujeres africanas en Situación de Trata: Diversidades, resistencias y oportunidades
}

\author{
African Women in Trafficking Situation: Diversities, resistances and \\ opportunities
}

\author{
Nuria Cordero Ramos \\ Universidad Pablo de Olavide \\ ncorram@upo.es \\ orcid.org/0000-0002-9875-1042 \\ Rafael Cáceres Feria \\ Universidad Pablo de Olavide \\ rcacfer@upo.es \\ orcid.org/0000-0002-9471-1211
}

\section{Resumen}

En este texto nos aproximamos a las mujeres africanas que se encuentran en una posible situación de trata con fines de explotación sexual. Nos proponemos analizar la complejidad de la trata de seres humanos (TSH), su fuerte vinculación con las políticas migratorias, y la relación simplificadora que a menudo se establece entre este fenómeno y la prostitución. Cuestionamos la utilización de la categoría "victima de trata" al considerarla una etiqueta estigmatizadora que niega la capacidad de toma de decisiones de estas mujeres, y destacamos un papel activo en la dirección de sus propias vidas. Por último, reflexionaremos sobre los problemas a los que estas migrantes se enfrentan, y cómo todos estos factores inciden en el modo en las instituciones públicas y privadas atienden sus necesidades. Visibilizamos a las mujeres africanas en situación de trata como sujetos de derecho frente a los discursos políticos y las prácticas asistenciales dominantes en la intervención social. 
Revisamos el rol de los agentes de intervención y resaltamos la necesidad del protagonismo de las propias mujeres durante estos procesos.

Palabras clave: Trata de Seres Humanos, Migraciones, Prostitución, Políticas Migratorias.

Abstract

In this text we approach African women who are in a possible situation of trafficking for the purpose of sexual exploitation. We propose to analyze the complexity of trafficking in human beings (TSH), its strong link with immigration policies, and the simplifying relationship that is often established between this phenomenon and prostitution. We question the use of the category "victim of trafficking" when considering it a stigmatizing label that denies the decision-making capacity of these women, and an active role in directing their own lives. Finally, we will reflect on the problems that these migrants face, and how all these factors affect the way in which public and private institutions attend to their needs.

We make African women in trafficking visible as subjects of law in the face of the dominant political discourses and welfare practices in social intervention. We review the role of intervention agents and highlight the need for the role of women themselves during these processes.

Key words: Trafficking Human, Migrations, Prostitution, Migration Policies 


\section{Introducción}

En este artículo nos aproximamos a las mujeres africanas que se encuentran en una posible situación de trata con fines de explotación sexual. Estamos ante un colectivo numeroso y diverso que, aunque comparte una situación de explotación ligada a factores estructurales (políticas migratorias, patriarcado, colonialismo...), presenta circunstancias muy variadas que hay que tener presente a la hora afrontar su estudio. Nos proponemos analizar la complejidad de la trata de seres humanos (TSH), su fuerte vinculación con las políticas migratorias, y la relación simplificadora que a menudo se establece entre este fenómeno y la prostitución. Por último, reflexionaremos sobre los problemas a los que estas mujeres se enfrentan, y cómo todos estos factores inciden en el modo en las instituciones públicas y privadas atienden sus necesidades.

En este trabajo ponemos en diálogo algunos de los aprendizajes provenientes del proyecto: Buenas prácticas en acción social con mujeres migrantes próximas a redes de trata en tránsito de Marruecos hacia Andalucía: fomentando la cooperación entre organizaciones de la sociedad civil, instituciones públicas y centros universitarios $\left(2015\right.$ SEC011) $^{1}$ (Cordero, Cáceres, Delgado y Embrane, 2018) y de los debates generados en las jornadas "Perspectivas sobre trabajo sexual y derecho" ${ }^{2}$ celebradas en la Universidad Pablo de Olavide (Sevilla) en diciembre de 2019.

Nos centraremos en cuatro aspectos específicos sobre este tema. En primer lugar, en el cuestionamiento de la utilización de la categoría "victima de trata", por considerarla una etiqueta que otorga a estas mujeres una identidad estática y pasiva; que opera estigmatizándolas, negándoles la capacidad de toma de decisiones y un papel activo en la dirección de sus propias vidas. Por ello, a lo largo del texto preferimos hablar de mujeres en situación de trata. Entendiendo que puede ser un hecho circunstancial, con frecuencia, ligado a proyectos migratorios, favorecido por las políticas fronterizas de Europa. Muchas de estas mujeres se ven abocadas a utilizar las "redes de trata" como una de las pocas posibilidades de atravesar las rutas que van de África a Europa. En segundo lugar, abordamos las diferentes formas de percibir la trata de seres humanos en los países de origen, tránsito y llegada. A pesar de que la concepción de este fenómeno y la preocupación por el mismo presenta importantes variaciones en países como Camerún, Marruecos o España, en los discursos internacionales se impone la mirada occidental, donde parece que el único interés se centra en la trata de mujeres con fines de explotación sexual, obviando otras formas de tráfico y explotación den personas. Un tercer aspecto sobre el que reflexionamos es la invisibilidad de la diversidad de estas mujeres. El estigma y los estereotipos que operan socialmente sobre las migrantes que están en prostitución terminan por difuminar todas sus diferencias, presentándolas de una manera homogeneizadora. A esto contribuyen los marcos legales que inciden en criminalizar la migración y la prostitución, y en encasillar a todas las africanas que están en prostitución bajo la

\footnotetext{
${ }^{1}$ Se trata ve una investigación financiada por la Agencia Andaluza de Cooperación Internacional para el Desarrollo (AACID). La finalidad del proyecto ha sido la de generar espacios de dialogo sobre las prácticas de intervención social destinadas a mujeres en situación de trata, tanto en los países de origen (Camerún) como en el tránsito (Marruecos) y en destino (Andalucía).

${ }^{2}$ Se trata de una actividad que forma parte del ciclo: Debates universitarios sobre trabajo sexual, celebrado en veinte universidades españolas, durante el curso 2019/2020. Se abordaba la realidad del trabajo sexual desde el ámbito académico.
}

Revista del Laboratorio Iberoamericano para el Estudio https://doi.org/10.46661/relies.XXXX 
etiqueta "víctima de trata". Estamos ante una categoría construida sobre una moral hegemónica que a las mujeres migrantes dedicadas a la prostitución como sumisas, engañadas, coaccionadas, negando tanto su diversidad como sus capacidades para tomar decisiones.

Por último, visibilizamos a las mujeres africanas en situación de trata como sujetos de derecho frente a los discursos políticos y las prácticas asistenciales dominantes en la intervención social. Revisamos el rol de agentes de intervención y resaltamos el protagonismo de las propias mujeres durante estos procesos.

\section{Mujeres africanas en situación de Trata de Seres Humanos.}

El conocido como Protocolo de Palermo (Naciones Unidas, 2000), conceptualiza la trata de seres humanos (TSH) como "la captación, el transporte, el traslado, la acogida o la recepción de personas, recurriendo a la amenaza o al uso de la fuerza u otras formas de coacción, al rapto, al fraude, al engaño, al abuso de poder de una situación de vulnerabilidad o a la concesión o recepción de pagos o beneficios para obtener el consentimiento de una persona que tenga autoridad sobre otra, con fines de explotación (Protocolo de Palermo 2000: Art.3). Por lo tanto, según esta definición, la TSH implica el desplazamiento dentro o fuera de un país, siendo los medios utilizados la coerción o la amenaza. En este sentido, entendemos que se puede establecer una relación directa entre las políticas migratorias restrictivas y las situaciones de violencia sobre los migrantes procedentes de países del Sur que deciden emigrar a Europa. La represión ante la llegada de migrantes está siendo la respuesta de las autoridades fronterizas y locales en los países de tránsito y destino para frenar los flujos migratorios. El endurecimiento de las políticas migratorias ha dejado a muchas personas en la pobreza y la exclusión ante la inaccesibilidad a los derechos fundamentales como seres humanos.

Por lo tanto, para aproximarnos a la realidad de las mujeres africanas que quieren llegar a Europa es necesario comprender los vínculos que existen entre "trata de seres humanos" y migración; primero por la relación que se deriva del movimiento de población de un país a otro, o dentro del mismo; y segundo, por los medios utilizados para la migración.

Las lógicas del mercado globalizado han provocado un aumento significativo, en países europeos, de la demanda de mano de obra femenina para las tareas relacionadas con el sector servicios, agrícola, trabajo doméstico y servicios sexuales. Estamos ante un mercado subalterno, marcado por el colonialismo y la desigualdad de género, que favorece una economía sumergida, poco regulada y mal remunerada (Vega, 2009). Este es el caso de numerosas mujeres africanas que se dedican a la prostitución en toda Andalucía.

La pobreza, la falta de oportunidades y la violencia de género (abusos sexuales y violencia intrafamiliar), son apuntadas por Women`s Link Worldwide (2014), como algunas de las principales razones que impulsan a numerosas africanas a emigrar a Europa. Para muchas de ellas, la decisión de abandonar su tierra se presenta como la única alternativa de mejorar sus condiciones de vida o/y la de sus familias. Esta decisión las impulsa a buscar opciones para preparar el viaje de acuerdo con sus posibilidades económicas, redes de apoyo locales y contactos en los países de destino. La finalidad es la de conseguir un trabajo que les permita lograr los ingresos necesarios para mantenerse $y$, además, enviar dinero a sus familiares que residen en sus países de origen.

Como reacción a los obstáculos que van a encontrar en este camino a Europa, producto de las políticas restrictivas sobre migraciones, se recurre a las "redes de trata" que se convierten en una plataforma para salir de su tierra; en otras ocasiones es en el tránsito desde el país de origen al de destino cuando muchas de estas mujeres contactan con estas redes, buscando la protección para realizar este trayecto. 
Desde los países occidentales esta situación se analiza de manera global sin tener en cuenta que estas migraciones tienen particularidades en función de las características de los países de procedencia, de las diferencias culturales y, sobre todo, de la población afectada. Unas veces, estamos ante decisiones individuales, y otras ante estrategias colectivas. Dependiendo de sus contextos de origen y de sus circunstancias, las mujeres utilizan unos medios u otros para el desplazamiento.

Sorprende la contradicción entre la dureza de las políticas migratorias europeas y la aparente preocupación por la trata seres humanos. En Europa los discursos políticos y de los medios de comunicación denuncian constantemente este fenómeno, reduciéndolo exclusivamente al tráfico de mujeres con fines de explotación sexual, y equiparándolo a prostitución. Muy poca atención se le presta, en cambio, a otras formas de trata que están igualmente presentes en los países occidentales. Muy distinto es el modo de percibir y abordar esta cuestión en los estados de origen y de tránsito. Tal como señala el informe Women's Link Worldwide (2014), en el caso concreto de Nigeria, uno de los países de donde proceden el mayor número de africanas que llegan a Europa, son las regiones del sur y, concretamente, el territorio del Edo State, cuya capital es Benin City, las zonas de donde parten más mujeres. En dicho estudio se señalan como el caldo de cultivo para que exista una construcción social normalizada en torno a la trata de personas: las altas tasas de corrupción, las graves desigualdades de género, una estructura social basada en la relación clientepatrón, así como una escasa protección de los derechos humanos y un destacado papel de la religión. Se podría decir que la trata se concibe, en algunas regiones de Nigeria, como una coyuntura a partir de la cual muchas familias ven en el envío de las mujeres jóvenes a Europa la oportunidad de mejorar sus condiciones de vida. "Otra característica de la trata de mujeres nigerianas con fines de explotación sexual es que éstas viven el proceso migratorio como algo más dañino que la explotación sexual en sí misma" (Movimiento por la Paz, 2019:12). Las graves vulneraciones de derechos que sufren durante el tránsito ponen de manifiesto la desprotección y la violencia a la que se encuentran sometidas las mujeres nigerianas que emigran a Europa.

En Camerún, un país en el que el número de mujeres migrantes hacia Europa va en aumento, la TSH y migración van de la mano. En este estado africano la trata cuando afecta a mujeres es considerada como una estrategia de migración, uno de los modos del salir del país, por lo que no se criminaliza (Alpes, 2012). Se entiende que es la consecuencia de una elección, unas veces individuales y otras familiares (Fischer, 2007). En cambio, existe mucha más preocupación por otras modalidades de trata: la explotación económica de cameruneses y camerunesas en los países del Golfo, así como la explotación interna de niños y niñas procedentes de áreas rurales de la zona norte (Kah, 2016), que es reconocida como un grave problema por parte de la sociedad camerunesa. Existen importantes diferencias en el modo de percibir este fenómeno según las regiones. Dentro del mimo país las estrategias, los canales de emigración y los países de destino varían según procedan las mujeres de las regiones anglófonas o francófonas.

Muy diferente es la situación de la "trata de seres humanos" en Marruecos, un país de emigración, donde la llegada de población africana camino de Europa ha supuesto el acercamiento a una realidad desconocida: la inmigración. La política de la Unión Europea promoverá que Marruecos se convierta en dique de contención de la frontera sur de Europa y será en ese momento cuando irrumpa esta cuestión en la agenda política de este país. La escasa concienciación social que hay en sobre este tema contrasta con el hecho de que exista una ley de trata, cuando muchos estados europeos no la tienen. En Marruecos la trata de personas se percibe de manera muy diferente a Europa. En primer lugar, no se ha focalizado en la explotación sexual de las mujeres. Entre otras razones porque oficialmente no se reconoce la existencia de prostitución. Sin embargo, existe un tráfico de niñas del campo a la ciudad, las denominadas petite bonne. Muchas familias ceden a sus hijas a un intermediario para que le busquen trabajo como empleada de hogar (Ajaaouani, 2013). 
Trabajan diariamente en situación de aislamiento, con salarios míseros y abusos verbales, físicos... y sin acceso a la educación (Llorent, 2013). Internamente se visibiliza más la explotación de estas menores que la de las mujeres que son objeto de la trata internacional. Igualmente, se le da más visibilidad a las mujeres tratadas procedentes de otros países africanos que a las marroquíes que son explotadas en otros países, a pesar de que en Marruecos, la ley 27/14 contra la "trata de seres humanos" protege a las mujeres al margen de su nacionalidad. Las inmigrantes que no han sido "víctimas de trata" son expulsadas, y ellas son conscientes de este hecho, al igual que las "redes de trata".

La forma de vincularse a las redes de trata, bien para realizar una parte del trayecto o el trayecto completo, provoca que las mujeres no se sientan identificadas como "víctimas". La aplicación de esta categoría es propia de los discursos políticos y de las prácticas sociales de los países europeos. Compartimos con Mansur (2017: 8) la idea de que "la celebración de una víctima genérica - carente incluso de historia - elimina la autonomía de los individuos, convirtiéndolos en seres inertes y sin agencia". En los casos analizados, las mujeres viajan a Europa por la intermediación de personas de su entorno, que les ofrece una posibilidad de afrontar el viaje, el engaño puede estar presente, para ocultar las dificultades de la travesía, el pago de las deudas y las duras condiciones laborales en los países de tránsito y/o destino. Sin embargo, son también significativas los casos de mujeres africanas que deciden emprender este viaje vinculadas a redes de tráfico de migrantes a las que se paga por hacer una parte o todo el trayecto, teniendo libertad para finalizar la relación con los intermediarios una vez que se ha logrado llegar al destino pactado. Concretamente, las mujeres que llegan a Andalucía por la costa no responden a un perfil estandarizado, si bien podemos señalar algunas características comunes:

-Proceden mayoritariamente de un número limitado de países africanos: Nigeria, Camerún, Mali, Guinea...

- Llegan a la frontera sur (Ceuta/ Melilla) después de duras travesías en las que, según reconocen, la posibilidad de supervivencia para las mujeres solas es mínima. Algunas inician el viaje vinculadas a redes de tráfico de personas o de trata; en cambio, otras son captadas en el trayecto y comienzan a ser explotadas sexualmente durante el tránsito (Ríos, 2016).

- Durante los trayectos sufren, junto con las duras condiciones de los traslados, la violencia de los controles fronterizos y amenazas y agresiones físicas y psicológicas por parte de los cuerpos y fuerzas de seguridad de los distintos países por los que pasan, embarazos, contagio de VIH y otras enfermedades de trasmisión sexual.

- Muchas de estas mujeres generarán lazos con estas redes y continuarán vinculadas a ellas de distinta forma: captando a otras mujeres, vigilando, apoyando a los hijos...

- Un número considerable de ellas dice no estar ligadas a la prostitución en sus países de origen, aunque algunas reconocen saber que tendrían que realizar trabajo sexual en Europa.

Por todo ello entendemos que es preferible hablar de mujeres en situación de trata; en primer lugar porque las propias mujeres no se identifican con esta categoría que opera en la intervención social, fruto de la aplicación de las políticas públicas vigentes en países de nuestro entorno occidental. En segundo lugar, porque en la mayoría de los casos estudiados, la vinculación con las redes forma parte, como venimos señalando, del propio proceso migratorio. Proceso condicionado por las políticas migratorias que imponen con dureza sus condiciones, condenando a la clandestinidad y a la lucha por supervivencia de las africanas que logran llegar a Europa. Los constantes cambios de las trayectorias vitales de estas mujeres, sin duda alguna pone en cuestión el estatismo que otorga la categoría de "víctima", al entender que existe un dinamismo propio de la paradoja entre 
vulnerabilidad y resistencia (Butler, 2001) que atraviesa la diversidad de situaciones que experimentan.

\section{Diversidades frente a la imposición del estigma de víctima de trata}

Tal como ha sido señalado, algunas de las mujeres africanas que están en situación de trata en Andalucía, las encontramos vinculadas a la prostitución. El elevado número de nigerianas en las redes europeas de prostitución las ha convertido, para los cuerpos y fuerzas de seguridad, en la cara más visible de la trata y la inmigración (Movimiento por la Paz, 2019). Esta es una de las razones por las que, con frecuencia, las africanas que están en Europa pasan a ser percibidas como prostitutas, y son consideradas genéricamente como nigerianas, o a lo sumo subsaharianas, un calificativo que tal como señala Herbert Ekwe-Ekwe (2010) es fruto de una visión colonialista de África. Esta mirada simplificadora sirve para homogenizar a todas mujeres africanas por el color de la piel y la pobreza, ocultando la diversidad cultural que existe no sólo entre las diferentes regiones del continente africano sino, también, dentro de los distintos países que lo componen. Concretamente Nigeria, es un ejemplo significativo de esa diversidad cultural: con más de cincuenta lenguas, numerosos grupos étnicos... Es un país donde las diferencias religiosas, étnicas y económicas son una fuente de conflicto social. Otro estado africano, Camerún, de donde proceden un creciente número de mujeres en situación de trata, aunque con una extensión mucho menor y una población menos numerosa, muestra la misma pluralidad étnica, lingüística, religiosa.... Las zonas norte y sur de Camerún, anglófonas, con mayoría de población musulmana, presenta unas circunstancias económicas más precarias, una gran inestabilidad política y una mayor violencia institucional.

Dada esta gran variabilidad, resulta pertinente reconocer la diversidad de edad, origen, etnia, religión, nivel educativo... (Brennan, 2005) que atraviesan a las mujeres africanas que están en prostitución, y dejar de considerarlas como un colectivo homogéneo. Ya que si bien es posible encontrar elementos comunes entre ellas, no es menos cierto que se suelen caracterizar a través de una mirada etnocéntrica que las percibe: pobres, analfabetas, supersticiosas, dependientes... proclives a ser engañadas y sin capacidad de agencia. En este sentido, se ha convertido en un lugar común afirmar que es frecuente que las "subsaharianas" en situación de trata sean controladas a través de la religión. No negamos que numerosas nigerianas de determinadas zonas del país sean amenazadas a través del uso de la magia, del vudú, del conocido como "yuyú" para ser explotadas, pero no hay que olvidar que estas ideas no son consustanciales a toda África. En los talleres realizados en Yaundé (Camerún) hemos podido comprobar que estas creencias no son compartidas por todas las africanas que migran. El contexto económico, el nivel educativo, la religión... son sumamente variables, incluso dentro de un mismo estado.

Se ha impuesto un discurso hegemónico que no tiene en cuenta que el género, la "raza", la etnicidad o la nacionalidad complejizan considerablemente este fenómeno (Zheng, 2010). Reconocer la diversidad de las mujeres que están Europa en situación de trata es un paso necesario, no solo para superar una mirada victimizadora sino también para entender sus circunstancias y brindarles el apoyo que requieren. Estas mujeres no solo proceden de culturas y contextos sociopolíticos heterogéneos, sino que poseen experiencias migratorias distintas que las lleva a tener una percepción muy diferente de su realidad, lo que exige una atención individualizada y sin aplicación de estereotipos.

Esta forma de homogeneizar a las mujeres negras migrantes no es exclusiva de Europa, también opera en Marruecos, donde encontramos los mismos clichés sobre las africanas procedentes de países del centro y el sur del continente. 


\section{Migración, trata y prostitución}

En Andalucía, muchas de estas mujeres camerunesas y nigerianas se dedican a la prostitución. Lo que significa que además de ser migrantes en situación de ilegalidad, se ven afectadas por el no reconocimiento de la actividad sexual como trabajo, y el estigma social que arrastra " la categoría de puta" (Osborne, 2004 ). Los discursos abolicionistas imperantes insisten en denunciar la utilización de los cuerpos de estas mujeres en el mercado del sexo, restándole importancia a otras situaciones de vulnerabilidad y explotación, como es la laboral, generadas por el sistema capitalista. Esta criminalización de la industria del sexo se potencia "a través de la intervención principal del derecho penal con la tipificación expresa de determinadas conductas que afectan de forma directa o indirecta a la práctica totalidad de los actores sociales" (López, 2010: 378). Estas percepciones dominantes quedan patentes con la puesta en marcha de leyes y normativas que regulan para que la prostitución sea erradicada para garantizar un orden moral hegemónico, utilizando para ello el enfoque trafiquista (Azize ,2004).

En la actualidad, cualquier debate sobre trata de seres humanos termina convirtiéndose en una disputa sobre la necesidad de legalizar o perseguir con mayor dureza el comercio sexual. En este sentido, el sociólogo Ronald Weitzer (2006) habla de una cruzada moral contra la prostitución y la trata con fines sexuales.

La asimilación entre trata y prostitución (a través de la puesta en marchas de los Planes Integrales y Normativas Municipales) que inunda las políticas públicas en España está generando un rechazo directo hacia comercio sexual, generando confusión, tanto en la ciudadanía como en las organizaciones dedicadas a la intervención social. Por ejemplo, las ordenanzas de Málaga sancionan a las personas que ejercen la prostitución, mientras que, en el caso de la de Sevilla, aunque no se las penaliza se ha creado una campaña contra el consumo de prostitución callejera atacando a los clientes, que "se ha traducido en un incremento de la presión policial y la represión de la actividad de las trabajadoras sexuales" (Acien, 2019:11). Esto ha provocado el ocultamiento de aquellas mujeres que ejercían en la calle, que han tenido que trasladarse a polígonos y lugares más alejados, en una situación de mucho más peligro e indefensión.

Al mismo tiempo, desde estos marcos reguladores se potencia una imagen victimista de las mujeres migrantes africanas, que se relaciona con una visión simplificadora de la realidad, que "es incapaz de ver actores /sujetos, seres, individuos; es incapaz de ver que lo esencial ocurre en las interretroacciones" (Morin, 1984: 355).

A modo de resumen, se podría decir que las políticas públicas contra la trata en Andalucía, y en general en toda España, son asimilacionistas, es decir, centradas en un modelo hegemónico que considera a las mujeres migrantes que están en prostitución como personas subalternas, cargadas de estigmas y sin capacidad de decisión sobre sus vidas. Sobre estas premisas ideológicas se va generando un imaginario cargado de clichés:

-Las mujeres africanas que están en prostitución sufren la desigualdad de género y no son responsables de sus decisiones sino más bien "víctimas que hay que salvar". Se considera que son mujeres, pobres y negras que han sido engañadas para venir a Europa.

-Los hombres consumidores de prostitución, así como todas las personas intermediarias que favorecen el ejercicio de esta actividad, son los causantes de esta desigualdad y, por lo tanto, han de ser sancionados y condenados.

- El ejercicio de la prostitución tiene que ser invisible, hay que alejarlo de los espacios públicos de las ciudades ya que altera el orden moral. Las mujeres africanas se ven obligadas a ejercer esta actividad en polígonos o carreteras alejadas de los centros urbanos (como es el caso de Sevilla) exponiéndolas a más riesgos y a la invisibilidad. 
La dualidad dominante en los discursos y prácticas de las instituciones en Andalucía, entre criminalización y victimismo del trabajo sexual y de la migración irregular, anula a las mujeres africanas como sujetos de derechos, para convertirlas en objeto de la intervención social de las diversas organizaciones que se dedican tanto a la atención de mujeres migrantes como de mujeres en contextos de exclusión. Desde la intervención social se insiste en la necesidad de (la) autoidentificación como víctima. Si no se identifican ellas mismas como víctimas (Alcazar y Cabeza, 2017) o no lo hacen los profesionales, las mujeres africanas que están en prostitución se ven excluidas del acceso a los derechos y las garantías que ofrece el sistema jurídico en nuestro país. En definitiva, los marcos legales no defienden los derechos humanos de estas mujeres (Cordero, 2014) sino que potencian el mal-trato, al insistir en el sometimiento durante el ejercicio de la prostitución, dejando de lado otras vulneraciones (de) derechos, e invisibilizando las capacidades y estrategias de las africanas que llegan a Andalucía.

\section{Resistencias y oportunidades}

Son numerosos los obstáculos y vulneraciones de derechos a los que se enfrentan las mujeres africanas que están trabajando en prostitución en Andalucía. Sin embargo, muchas de ellas no son mujeres "traídas" y "tratadas" sino que, como se ha venido exponiendo; son personas que, en su mayoría, tienen un proyecto migratorio con finalidades diversas según sus propias circunstancias personales, familiares, sociales y culturales. De nuestros aprendizajes en la praxis partimos del convencimiento de que son numerosas las africanas que están en prostitución con capacidad de agencia. Estamos de acuerdo con Sen (1995: 71) cuando afirma que "una persona como agente no tiene por qué guiarse solamente por su propio bienestar". Puesto que puede proponerse metas sociales que no les proporcionan necesariamente bienestar personal a ellas directamente. Esta capacidad de agencia la consideramos como la aptitud de crear acciones y tomar decisiones teniendo en cuenta todo el entramado de relaciones que rodean cada circunstancia.

En el caso de las mujeres que inician un proyecto migratorio con la finalidad de llegar a Europa y que están en prostitución en Andalucía "lo que está claro es que la mayoría decidieron de forma autónoma que quedarse en sus países de origen no era la mejor forma de sacar adelante a su familia o desarrollar una vida próspera." (Acien, 2010).

El trabajo de campo nos muestra que la prostitución, para muchas mujeres, es una estrategia para lograr sus finalidades y que, con frecuencia, conocían antes de emprender el viaje, que, probablemente, tendrían que dedicarse a esta actividad. Lo que la mayoría afirman es que desconocían las condiciones de explotación a las que se verían sometidas, y que son invisibilizadas, tanto por los discursos políticos como por las prácticas de intervención. El trabajo sexual es una estrategia económica (Ríos, 2016) que permite a muchas africanas ayudar a sus familias y ascender en la categoría social con respecto a otras mujeres de sus países de origen. El no reconocimiento o la negación de las estrategias de supervivencia que emplean estas migrantes contribuyen a reforzar el carácter asistencialista, fomentando, en palabras de Aradau (2004), "políticas de la lástima".

En Andalucía estas mujeres están el punto de mira de instituciones públicas y privadas, siendo atendidas por organizaciones de la sociedad civil dedicadas a la intervención con mujeres en situación de exclusión y/o a migrantes. Se convierten así en usuarias de programas de intervención social destinados a las "víctimas de trata" o víctimas de explotación sexual.

La provisionalidad de los programas, la burocratización, la prioridad de responder a necesidades básicas, así como el escaso compromiso de los poderes públicos, son algunos de los obstáculos a los que se enfrentan diariamente profesionales y mujeres migrantes. La escasa dotación de recursos públicos y la falta de garantías reales para enfrentarse a la vulneración de derechos que sufren en Andalucía estas mujeres, impiden que desde la intervención social se puedan dar respuestas 
integrales a cada situación. Esto provoca que las mujeres continúen sometidas a lo que Young describe como opresión y dominación:

"consiste en procesos sociales institucionalizados que anulan la capacidad de las personas para interactuar y comunicarse con otras o para expresar sus pensamientos y perspectivas sobre la vida social en contextos donde otras personas pueden escucharlas." (Young, 2000: 68).

La opresión se va perpetuando a través de un sistema jerárquico de intervención basado en la burocracia y en un modelo abstracto de "victima de trata "que niega las realidades concretas de cada una de las mujeres. El desconocimiento de las realidades particulares y la burocracia institucional generan distanciamiento y falta de confianza hacia profesionales de la intervención social. Consideramos que algunos procedimientos como interrogar reiteradamente en cada entrevista sobre las mismas cuestiones: proceso migratorio, por qué han venido, de qué forma lo han conseguido, desde cuando están en prostitución, quienes son las personas que las explotan...resulta re-victimizante. En numerosas ocasiones se les solicita que denuncien a sus parejas o personas de confianza para poder prestarles ayuda, no teniendo en cuenta sus vínculos con compañeros, familiares de origen y personas intermediarias, según sus propias claves culturales. Todo esto pone de manifiesto que la protección de las mujeres, por parte de estado español, no debe estar condicionada a la colaboración con la justicia.

Nos parece necesario revisar el rol asumido por los agentes de la intervención, pues algunas prácticas que apuntan a la transformación social y el bienestar, sin proponérselo, pueden estar permeadas por gestos orientados al control de las poblaciones, la individualización de los problemas sociales y la administración de las desigualdades para garantizar el mantenimiento del status quo (Moreno y Molina 2018).

La finalidad de la intervención social con las mujeres migrantes en situación de trata ha de estar orientada a acompañarlas en el proceso migratorio, más allá del control sobre su actividad o sobre sus hijxs, sin violentar sus decisiones y evitando revictimizarlas. Una intervención, a medio y largo plazo, que vendría marcada por las "contingencias de los encuentros" (Moreno y Molina 2018) y el reconocimiento de las propias mujeres como agentes de sus proyectos vitales.

Siguiendo la propuesta de Young (2000) planteamos estrategias de intervención social que estén orientadas a fomentar el autodesarrollo y la autodeterminación. Autodesarrollo, entendido como un ejercicio de desarrollo de las capacidades singulares y propias que cada mujer posee, y que tiene el derecho de expresar como un rasgo de su identidad, como por ejemplo expresar sus creencias religiosas, compartir sus conocimientos y prácticas de autocuidado y dar a conocer sus modelos familiares sin sentirse juzgadas. Autodeterminación a través de la participación activa, articulada por medio de las decisiones y determinaciones que cada mujer asume como ciudadana. Esto supone generar procesos de intervención y de comunicaciones horizontales y democráticas que fomenten que las propias mujeres sean las que realicen incidencia política para revertir las condiciones de opresión que están viviendo.

Los procesos de IS deben re-conocer los obstáculos que estas migrantes han superado en los lugares de origen, durante los tránsitos migratorios y en los países de destino, con la finalidad de visibilizar sus demandas, estrategias de supervivencia y capacidades. Siguiendo a Martinelli (2011) podemos afirmar que es en la cotidianeidad de las personas y los grupos donde residen buena parte de los actos de resistencia ante las violaciones de derechos. Potenciar las "capacidades centrales" (Nussbaum, 2012) en lugar de la victimización ofrece la oportunidad de revertir el estigma generado por la migración y prostitución y abrir espacios para la revalorización, reconocimiento y recreación de otras identidades.

Esta forma de proceder en la intervención social ofrece el acompañamiento y la horizontalidad frente al modelo dominante vertical-burocrático-asistencial de interventor-intervenido que Revista del Laboratorio Iberoamericano para el Estudio Sociohistórico de las Sexualidades https://doi.org/10.46661/relies.5103 
intentan reproducir los mandatos sesgados de las políticas dominantes. Las narraciones en primera persona, de las mujeres migrantes muestran las propias contradicciones de este modelo dominante:

"El discurso y las prácticas de la otredad también han definido a las mujeres inmigrantes en relación con un cierto ideal de emancipación femenina que es, con frecuencia, el modelo de la mujer occidental." (Mayorga, 2012: 57).

La instauración de un clima de confianza mutua, y el interés recíproco de conocer sus experiencias y demandas se convierte en oportunidad para ir tejiendo estrategias de movilización que logren el reconocimiento de sus derechos.

Se hace imprescindible un discurso político que reconozca a las mujeres migrantes como sujetos de derecho, que defienda sus condiciones frente a la explotación, y que garantice la dignidad a las mujeres africanas que están en situación de trata. Dignidad como principio del que se "desprende necesariamente la elección de políticas que protejan y apoyen la agencia (la capacidad de acción de cada sujeto) en vez de otras que infantilicen a las personas y las traten como receptoras pasivas de prestaciones" (Nussbaum, 2012:46). Los debates teóricos más propios del ámbito universitario deben ser retroalimentados con los discursos de las propias protagonistas con la finalidad de lograr procesos de intervención que sean potenciadores de lucha por la dignidad de las personas afectadas por la trata, a través del empoderamiento y el reconocimiento de las mujeres migrantes.

\section{Conclusiones}

Este texto es fruto de nuestra de colaboración con instituciones que trabajan con mujeres migrantes en situación de trata y, por lo tanto, deriva de nuestra experiencia directa con estas mujeres. No pretendemos presentar ningunas conclusiones definitivas sino, más bien, dar voz a muchas africanas de las que frecuentemente se habla, pero a las que pocas veces se las escucha. Nos limitamos a sintetizar nuestros aprendizajes, derivados de la realización de un proyecto de investigación, así como del debate sobre el trabajo sexual celebrado en la UPO:

1.- Numerosas mujeres africanas en situación de trata de seres humanos con las que hemos hablado no se identifican a sí mismas como víctimas. Esa categoría responde a un modelo de intervención, basado en la criminalización de la migración irregular y la prostitución. La etiqueta abstracta y etnocéntrica de "víctima de trata con fines de explotación sexual" se impone sobre las identidades particulares con el fin de ordenar, controlar y reproducir el orden social hegemónico que utiliza la realidad de estas migrantes para enmascarar a los verdaderos victimarios que son los gobiernos de los estados europeos.

2.-Muchas nigerianas y camerunesas quieren viajar a Europa con el objetivo de mejorar sus condiciones de vida y las de sus familias. Es un proyecto migratorio individual- familiar donde las redes de trata forman parte de una estrategia utilizada para salir de sus países de origen y llegar al continente europeo, o para realizar parte de ese trayecto. En algunos estados de Nigeria, las redes que envían mujeres a Europa están imbricadas en la estructura social del país. En el caso de Camerún parece que están más diluidas con las redes de tráfico de personas. La diversidad propia de cada estado la expresan las propias mujeres en la forma de entender la familia, la sexualidad, la maternidad, el trabajo, los vínculos sociales... Una vez que se embarcan en el proyecto migratorio, las identidades particulares están permeadas por las adaptaciones que les exige la propia supervivencia a lo largo de todo el trayecto.

3.- La meta de estas mujeres africanas es salir se sus países y llegar a Europa. El engaño y la coacción a los que se alude desde el enfoque trafiquista están distorsionados. Las principales presiones que las impulsan a marcharse de sus lugares de origen son un producto de las lógicas del modelo 
neoliberal, patriarcal y colonial que se concretan en las restricciones de las políticas migratorias. En los discursos occidentales el engaño se presenta como un desconocimiento sobre la dedicación al trabajo sexual en el destino, sin embargo, a lo que hacen referencia las protagonistas es a las adversas circunstancias que tienen que soportar durante su recorrido hasta Europa, como consecuencia de las restricciones de las políticas migratorias que están provocando. Hablan de las duras condiciones vividas en "el camino" y la dificultad de atravesar distintos puestos fronterizos. Según ellas es imposible que sobreviva una mujer sola sin ningún vínculo con las redes.

4.- En Andalucía y en el estado español, las situaciones que viven las mujeres africanas que están en prostitución son utilizadas en los discursos políticos para perpetuar un orden moral, donde las propias mujeres tienen que elegir entre el "estigma de puta o el "estigma de víctima". Esta dicotomía las hace elegir de nuevo la opción que ofrece más posibilidades para seguir adelante con sus proyectos migratorios. Las políticas públicas y las ordenanzas locales deberían promover los derechos de las mujeres africanas que están en prostitución, con el fin de ofrecer garantías dentro del sistema público para lograr, al menos, las mismas condiciones de ciudanía que las mujeres españolas.

5.- La mayoría de las organizaciones y entidades sociales que atienden a estas mujeres siguen mayoritariamente las directrices marcadas por las "políticas de lástima". Desde estas entidades se realiza la "detección" como víctima de trata, que es la puerta de entrada para optar a la ayuda institucional. Esto supone, en el mejor de los casos, tener la cobertura de algunas necesidades básicas. Consideramos que en los procesos de intervención social se debería de incluir la voz de las mujeres en esta situación para poder conocer sus demandas, con el fin de orientar los recursos de intervención de manera que respondan a sus necesidades. Estos procesos deberían reconocer y potenciar las capacidades de estas mujeres a partir de interacciones, desde modelos de relación menos burocratizados y generadores de relaciones de confianza. Proponemos que se podrían impulsar metodologías participativas, por ejemplo, utilizando técnicas artísticas que fomenten la expresión de sentimientos y el fortalecimiento grupal de manera más distendida. Al mismo tiempo, desde estas entidades se debe fomentar la revisión crítica del enfoque trafiquista, orientando la incidencia política a garantizar derechos de las mujeres migrantes que están en prostitución. Y desde la universidad, los debates teóricos deberían contemplar los puntos de vista de las protagonistas, para evitar caer en posiciones etnocéntricas y paternalistas. 


\section{Bibliografía}

Acién, E. (2010). Una experiencia de investigación etnográfica en el Poniente almeriense: Nigerianas en el trabajo sexual. Pagina Abierta, 206, 20-25. Recuperado de http://www.pensamientocritico.org/estaci0310.html

Acien, E. (2019). Sacudirse el estigma: Apropiación del término 'puta' desde el activismo por los derechos en el trabajo sexual. Gazeta de Antropología, 35(1), artículo 04. Recuperado de http://www.gazeta-antropologia.es/?p=5136

Ajaaouani, N. (2013). La realidad jurídica y social de les petites bonnes en Marruecos. En M. Cabrera Espinosa y J. A. López Cordero (Eds.), V Congreso Virtual sobre Historia de las Mujeres (pp. 15-31). Jaén: Archivo Histórico Diocesano.

Alcazar, A., y Cabeza, E. (2017). El paradigma discursivo en torno a la víctima de trata: Intervención social con mujeres dominicanas en Puerto Rico. Disparidades: Revista de Antropología, 72(1). 85102. doi: 10.3989/rdtp.2017.01.003.

Alpes, M. J. (2012). Bushfalling at all cost: The economy of migratory knowledge in Anglophone Cameroon. African Diaspora, 5(1), 90-115.

Aradau, C. (2004). The perverse politics of four letter words: Risk and pity in the securitisation of human trafficking. Journal of International Studie, 33(2), 251-277. doi: $10.1177 / 03058298040330020101$

Azize, Y. (2004). Empujar las fronteras: Mujeres y migración internacional desde America Latina y el Caribe". En R. Osborne (Ed.), Trabajador@s del Sexo (pp. 167-191). Barcelona, Bellaterra.

Brennan, D. (2005). Methodological challenges in research with trafficked persons: Tales from the field. Internacional Migration, 43(1-2), 35-54.

Butler, J. (2001). El género en disputa: El feminismo y subversión de la identidad. México: Universidad Autónoma de México.

Cordero, N. (2014). Trata con fines de explotación sexual: Derechos humanos que mal-tratan a las humanas. Gazeta de Antropología, 30(3), artículo 09. Recuperado de http://www.gazetaantropologia.es/?p=4627

Cordero, N., Cáceres, R., Delgado, J., y Embrane, Z. (2018). Decálogo de Buenas Prácticas para la acción social con mujeres migrantes en situación de trata durante el tránsito de África a Europa: Generando transferencia de conocimiento entre la universidad y las entidades sociales. Madrid: Dykinson

Ekwe-Ekwe, H. (2010, 13 de mayo). ¿Qué es esto de África subsahariana? Pueblos: Revista de Información y Debate. Recuperado de http://www.revistapueblos.org/old/spip.php?article1873

Kah, H. K. (2016). Child trafficking across the Cameroon / Nigeria border: A historical perspective. Covenant University Journal of Politics \& International Affairs, 4(1), 1-16.

Llorent, V. (2013). Las petites bonnes marroquíes: Causas y consecuencias socioeducativas. Revista Educatio Siglo XXI, 31(1), 335-356.

Revista del Laboratorio Iberoamericano para el Estudio https://doi.org/10.46661/relies.5103

Sociohistórico de las Sexualidades 
López Riopedre, J. (2010). Inmigración colombiana y brasileña y prostitución femenina en la ciudad de Lugo: Historias de vida de mujeres que ejercen la prostitución en pisos de contactos (Tesis doctoral). Universidad Nacional de Educación a Distancia, España. Recuperada de http://espacio.uned.es/fez/view.php?pid=tesisuned:CiencPolSoc-Jlopez

Mansur, G. (2017). Trata de personas, tráfico de migrantes y la gobernabilidad de la migración a través del crimen". Etnográfica, 21(3), 1-13.

Martinelli, M. L. (2011). O serviço social e a consolidação de direitos: Desafíos contemporáneos. Revista de Serviço Social \& Saúde. Sao Paulo, 10(12), 1-17.

Mayorga, C. (2012). Articulaciones de la exclusión: La política de atención a prostitutas inmigrantes en Madrid". Si Somos Americanos: Revista de Estudios Transfronterizos, 1, 49-74.

Moreno, M. A. y Molina, N. (2018). La intervención social como objeto de estudio: Discursos, prácticas, problematizaciones y propuestas. Athenea Digital: Revista de Pensamiento $e$ Investigación Social, 18(3), p. e-2055.

Morin, E. (1984). Ciencia con consciencia. Barcelona: Anthropos.

Movimiento por la Paz. (2019). La trata de mujeres hoy. Mujeres nigerianas víctimas de trata en España: Informe 2018. Recuperado de https://www.mpdl.org/noticias/global/derechoshumanos/informe-trata-mujeres-hoy-mujeres-nigerianas-victimas-trata-espana

Naciones Unidas. (2000). Protocolo para prevenir, reprimir y sancionar la trata de personas especialmente mujeres y niños, que complementa la Convención de las Naciones Unidas contra la Delincuencia Organizada Transnacional (Protocolo de Palermo). [Consultado 24 de noviembre de 2018]. Recuperado de http://www.ohchr.org/Documents/Professionallnterest/ProtocolTraffickingInPersons sp.pdf

Nussbaum, M. (2012). Crear capacidades. Propuesta para el desarrollo humano. Barcelona: Paidós.

Osborne, R. (2004). Introducción. En R. Osborne (Ed.), Trabajador@s del sexo. Derechos, migraciones y tráfico en el siglo XXI (pp. 11-40). Barcelona: Bellaterra.

Rios, A. (2016). Ocuparse en la industria del sexo: ¿Una estrategia de supervivencia frente a la crisis? Revista Internacional de Estudios Migratorios, 6(2), 269-291. Recuperado de http://www.riem.es/espanol/ContadorArticulo.php?idart=67

Sen, A. (1995). Nuevo examen de la desigualdad. Madrid: Alianza.

Vega, C. (2009) Culturas del cuidado en transición: Espacios, sujetos e imaginarios en una sociedad de migración. Madrid: UOC.

Weitzer, R. (2006). Moral crusade against prostitution. Society, 43(3), 33-38. 
Women's Link Worldwide. (2014). La trata de mujeres y niñas nigerianas: esclavitud entre fronteras y prejuicios. Madrid: Womens Link Worldwide. Recuperado de http://www.Irmcidii.org/wpcontent/uploads/2014/04/Women s Link Trata de mujeres y ni as africanas.pdf

Young, I. M. (2000). La justicia y la política de la diferencia. Valencia: Cátedra.

Zheng, T. (Ed.). (2010). Sex trafficking human rights and social justice. New York: Routledge. 